\title{
Synchrophasor measurement based damping estimation and oscillation mode detection using FFT-CWT approach in Japan campus WAMS
}

\author{
Khairudin $^{\mathrm{a}, \mathrm{b}}$, Yaser Qudaih ${ }^{\mathrm{a}}$, Masayuki Watanabe ${ }^{\mathrm{a}}$, Yasunori Mitani ${ }^{\mathrm{a} *}$ \\ ${ }^{a}$ Kyushu Institute of Technology, 1-1 Sensuicho, Kitakyushu 804-0015, Japan \\ ${ }^{b}$ Lampung University, Jl. Prof. S. Brojonegoro No. 1, Bandar Lampung 35145, Indonesia
}

\begin{abstract}
The low voltage level synchrophasor measurement data are investigated to estimate the damping ratio and to detect any oscillation mode using Fast Fourier Transform (FFT) and Continuous Wavelet Transform (CWT). Complex Morlet $(\mathrm{CM})$ is selected as a mother wavelet function considering its ability to reveal time and frequency localization simultaneously. A Two Area Four Machine System (TAFMS) model is elaborated using the Power System Analysis Toolbox (PSAT) and the FFT-CWT approach to convince the validity of the proposed method by comparing the two results. Finally, Phasor Measurement Unit (PMU) data from Japan Campus WAMS at some disturbances are evaluated.
\end{abstract}

Keywords: PMU, PSAT, FFT, CWT, damping ratio, oscil-lation mode, complex morlet function, small signal stability, two area four machine system

\section{Introduction}

The application of Phasor Measurement Unit (PMU) in power system has widely been used in the last decades. Since then many research works have been conducted and research topic in the field of security assessment, fault location detection, wide area protection, instability prediction and power system state estimation are widely opened [1]-[5].

Generally, a PMU is installed in substation or generation station. It measures magnitude and angle of current and voltage of the corresponding bus. This device essentially is the direct descendant of Symmetrical Component Distance Relay (SCDR), which is used for protecting high voltage transmission line [3]. The capability of this device to measures the phasor of voltage and current in a very high accuracy and synchronizes it using Global Positioning System (GPS) clock makes it possible to assess the system state or to locate a disturbance in the transmission line.

Recently, the deployment of PMU in a domestic network is beginning popular constituted by a variety of reasons. One of them is in Japan, where many different companies operate the power system; sharing data is an uneasy thing to do. Hence, the application of PMU in a campus scale where some universities working in collaboration in a Japan Campus WAMS is a reasonable choice.

Working with PMU data acquired from a domestic outlet is a fascinating challenge. All system data such as generators, transformers, tie lines and load parameters are all unknown. The analysis had to be carried out relying only on to the phasor signals. Therefore, a vigorous method is unavoidably needed. This research elaborates an alternative approach in dealing with PMU data to estimate damping ratio based on Fast Fourier Transform (FFT) and Continuous Wavelet Transform (CWT) where Complex Morlet Function is chosen as the mother wavelet then followed by demodulating the signal skeleton at a

\footnotetext{
* Manuscript received June 30, 2015; revised July 23, 2015.

Corresponding author. Tel.: 81-70-1949-1998; E-mail address: m589505b@mail.kyutech.jp.

doi: $10.12720 /$ sgce.4.2.93-102
} 
ridge and peak of the 3-D waveform.

The modulation signal is the signals envelope, which measures the dissipation of oscillation energy caused by damping. Therefore, the damping can be estimated by extracting the signal envelope using a particular de-modulation method. The Logarithmic and Phase decrement of the CWT Modulus are projected using linear regression method to extract the damping ratio from the signal. The calculations are purely based on information from the signal regardless to any network model assumption.

The cogency of this method is verified by comparing the result of damping ratio of Kundur Two Area Four Machine System (TAFM) using the Power System Analysis Toolbox (PSAT) to the outcome of this proposed method. The results indicate that both methods are about the same, which means that the FFTCWT method is potentially applicable to the practical uses.

Fig. 1 describes the process of identification of oscillation mode and calculation of damping ratio in this study. PMU Signal or simulated signal is transformed the Time-Frequency-Modulus representation using CWT where the center of oscillation resulted from the FFT calculation is employed as one of the wavelet parameters. On the other works [6]-[10] the center of oscillation is predicted using CWT itself. The advantage of using FFT to estimate the center of oscillation is its simplicity and clarity to break away each mode contained in the signal. This is the contribution of this work.

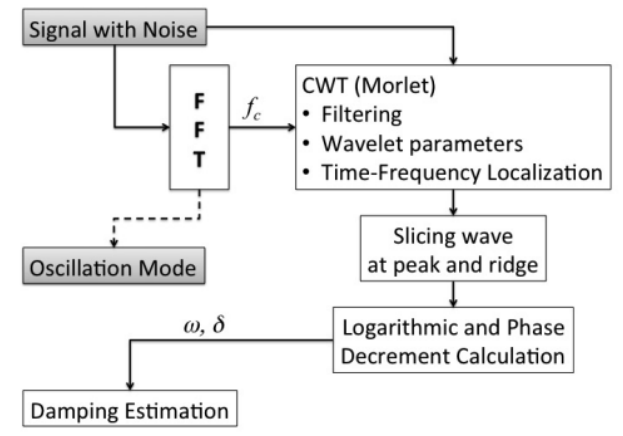

Fig. 1. Flow chart of calculation method.

\section{Synchrophasor Measurement in Japan Campus Wams}

Typically, two issues will come up when measuring the phasor using PMU in the low voltage network. First is that because of the PMU is fed up with a single-phase voltage then the measurement exhibits some slight error due to a constant sampling interval at off-nominal frequencies as well as on the high voltage measurement. The second is that local switching events in the low voltage network are also measured and might be interfused with oscillation mode.

Dealing with the two conditions above, some previous work in [11] and [12] had verified the validity of this measurement type. In [11] the single voltage phase difference from PMUs measurement at low voltage level were compared to the actual power flow of a $230 \mathrm{kV}$ tie line between center area and south area of Thailand power system. In [12] the phasor measurements from PMUs installed in $100 \mathrm{~V}$ power outlets at the central supply area of Kyushu, Kansai, and Chubu electric power company were compared to the measurement of PMU in the $500 \mathrm{kV}$ power station at west end of Chubu, PMU located in the 500 $\mathrm{kV}$ power stations at east end and PMUs which are installed in the $275 \mathrm{kV}$ generating stations. The two investigations above demonstrate the strong correlation between two sets of data confirming the validity of the phase difference measurement at low voltage level. Those results indicate $100 \mathrm{~V}$ power outlet phasor properly reflects the oscillatory characteristics of upper power system including the power outlet. Additionally, as for second issue, the influence caused by relative high frequency low voltage switching events could be eliminated by applying signal filtering method [13], [14].

In Japan Campus WAMS, PMUs are installed in thirteen universities as shown in Fig. 2. The idea 
behind of nurturing this Campus WAMS is because of Japan power system run by multiple companies. Each of those power companies is an independent operating unit hence collecting the synchronized phasor measurements data of transmission high voltage level from all power companies is something uneasy at present. Through the Campus WAMS it becomes possible to observe and analyze system dynamics of overall Japan nationwide grid system without bothering the power companies. All PMUs measure the single phase voltage phasor of $100 \mathrm{~V}$ on the laboratory wall outlet with GPS-synchronized time tag. The voltage phasor is acquired by using 96 sample data per voltage sine-wave cycle. The data is saved hourly in PMU at a certain interval of time [11], [15]. The phasor measurements from all locations are automatically transferred to the data server through the Internet.

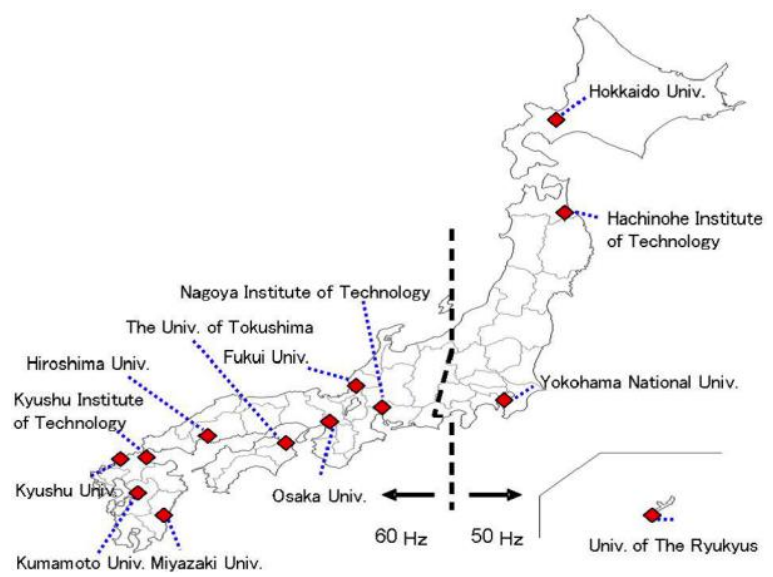

Fig. 2. PMU location in Japan Campus WAMS.

\section{The Application of FFT-CWT Approach}

\subsection{Oscillation mode identification using FFT}

The Oscillation mode of a set of signal can be easily revealed using spectral analysis. Taking advantage of the well-known mathematical function FFT that can transform a signal from the time domain to the frequency domain, it is possible to identify the frequencies concealed in a signal. The FFT is a numerical algorithm that is commonly used to compute the Discrete Fourier Transform (DFT) of an n-dimensional signal in a very efficient time compared to other methods such as solving simultaneous linear equation or the correlation method [16], [17]. In this work, the oscillation modes are discovered using FFT by separating each dominant frequency contained in a composite signal.

In fact, there are some other techniques to recognize the mode of set of signals, from the classical Prony Analysis to the relatively new method Hilbert Huang Transform (HHT) as it has been compared in [18]. Prony analysis is more suitable for stationary signal, therefore a window signal where the system does not change so much should be selected to get an accurate result as discussed in [18]. HHT is capable of estimating modal parameters, still it cannot distinguish two split up modes unless there is a large difference in either frequency or damping ratio [8], [18].

To demonstrate the ability of the FFT method in recognizing modes from a set of signals, a synthesized signal in time domain in (1) is converted to frequency domain using FFT algorithm to identify the center of oscillation. The parameter values of individual mode are given in Table 1. A random noise signal $r(t)$ represents local switching and other noises in the real PMU signal. The amplitude of the noise is selected so that not bigger than the amplitude of the ringdown signal in order not burying off the actual signal we need to analyze.

$$
f(t)=r(t)+\sum_{i=1}^{3} A_{i} e^{-2 \pi \zeta t} \cos \left(2 \pi f_{i} t+\theta_{i}\right)
$$


Table 1. Parameter value of each signal mode

\begin{tabular}{ccccc}
\hline $\begin{array}{c}\text { Mode } \\
(i)\end{array}$ & $\begin{array}{c}\text { Amplitude } \\
(A)\end{array}$ & $\begin{array}{c}\text { Frequency } \\
(f)\end{array}$ & $\begin{array}{c}\text { Damping } \\
(\zeta)\end{array}$ & $\begin{array}{c}\text { Shift Angle } \\
(\theta)\end{array}$ \\
\hline 1 & 1.0 & 0.35 & 0.26 & $0^{0}$ \\
2 & 0.5 & 0.50 & 0.50 & $30^{\circ}$ \\
3 & 0.9 & 0.90 & 0.11 & $60^{\circ}$ \\
\hline
\end{tabular}

Table 2. Real and estimated center of oscillation value of each signal mode

\begin{tabular}{|c|c|c|c|}
\hline Mode & \multicolumn{2}{|c|}{ Center of Oscillation } & Error Rate \\
\hline (i) & Real & Estimated & $(\%)$ \\
\hline 1 & 0.35 & 0.350 & 0 \\
\hline 2 & 0.50 & 0.500 & 0 \\
\hline 3 & 0.90 & 0.900 & 0 \\
\hline
\end{tabular}

The experimental signal in time domain is shown in Fig. 3(a) and the result of estimation using this FFT Algorithm is depicted in Fig. 3(b). The comparison between the given and the estimated value are presented in Table 2. It confirms this approach is pertinent to identify the mode contained in a set of synthesized signal.

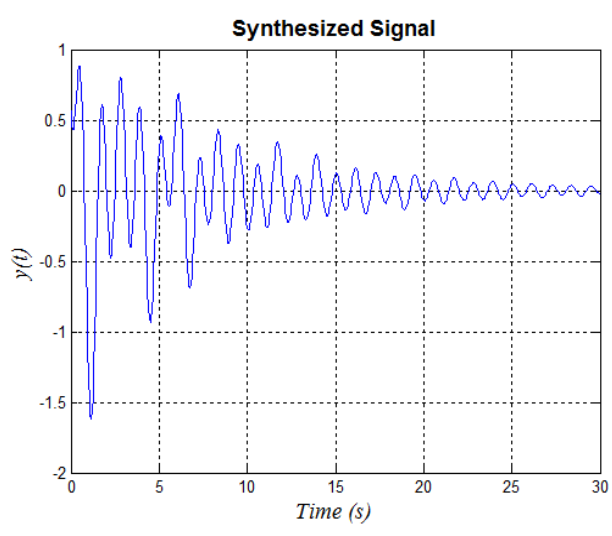

(a)

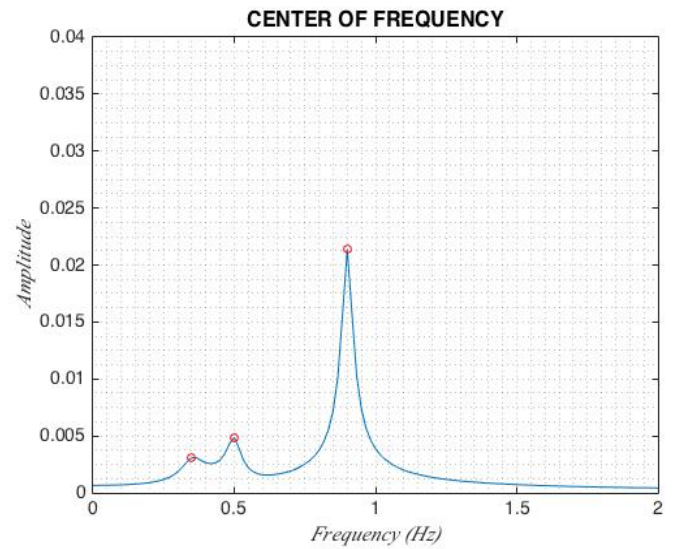

(b)

Fig. 3. The synthesized signal contains of three modes (a) and Centre of frequency of each signal mode (b).

\subsection{Continuous wavelet transform}

The wavelet transform decomposes signals over dilated and translated wavelets. A wavelet is a function of $f(t) \in L^{2}(R)$ with a zero average. It has been proved that the CWT is very dexterous to identify the damping ratio and shown to be highly resistant to noise, i.e. of up to $0 \mathrm{~dB}$ signal-to-noise ratio [6], [7].

Suppose that all function $f(t)$ satisfy the condition:

$$
\int_{-\infty}^{+\infty}|f(t)|^{2} d t \leq \infty
$$

Then, the CWT of the signal can be defined as:

$$
W f(u, s)=\int_{-\infty}^{+\infty} f(t) \psi_{u, s}^{*}(t) d t
$$

where $u$ and $s$ are the translation and the scale parameters respectively. $\psi^{*}{ }_{u, s}(t)$ is the translated and scaled complex conjugate of the mother wavelet function $f(t) \in L^{2}(R)$. The wavelet function is a normalized function i.e. the norm is equal to one with an average of zero [19].

The scaled-and-translated wavelet function is expressed as: 


$$
W_{(u, s)}(t)=\frac{1}{\sqrt{s}} \psi\left(\frac{t-u}{s}\right)
$$

It can be noted that the CWT is the sum over all time of the signal $f(t)$ multiplied by scaled, shifted versions of the mother wavelet (which are also called son wavelets). Thus, the CWT possesses localization properties in both time and frequency domains and consequently provides valuable information about $f(t)$ at different levels of resolution and measures the similarity between and each son wavelet [8].

\subsection{Complex morlet function}

The admissibility condition implies $\psi(0)=0$, which means that a wavelet must integrate to zero. In some literature, it is mentioned that there are several different types of mother wavelet functions satisfying the admissibility condition such as the Mexican Hat, Gabor and Morlet, which can be selected according to the nature of the signal to be analyzed. According to those literatures the Complex Morlet Wavelet Function (CMWF) would be appropriate for the analysis of ring down signals due to its capabilities in time-frequency localization for analytical signals [7]-[9], [20]. According to [19], the CMWF is capable of analyzing data in a multi-resolution domain which means it can automatically filter out the noise from $f(t)$ and thus no additional filters are needed.

Following the work on [7], [8], [19], the CWT of a continuous time signal can be expressed by the inner product of the Hilbert Space as follows:

$$
W_{h} f(a, b)=\frac{1}{\sqrt{a}} \int_{-\infty}^{+\infty} f(t) h^{*} \frac{(t-a)}{b} d t
$$

where $\boldsymbol{a}$ is a dilation or scaling factor, $\boldsymbol{b}$ is a translation or time shift factor, and $h^{*}(t)$ is complex conjugate of a mother wavelet function $h(t)$.

Again, taking advantage of the work on [7], [8], The Complex Morlet Wavelet Function is selected and formulated as:

$$
h(t)=e^{j 2 \pi f_{c} t} e^{\frac{-t^{2}}{f_{b}}}
$$

$f_{c}$ is the center of oscillation and $f_{b}$ is bandwidth frequency parameters.

Let's have a look at a function of a ring down type signal in (7):

$$
x(t)=a e^{-\alpha t} \cos (\omega t+\theta)
$$

that can be re-written as:

$$
x(t)=A(t)\left|\frac{e^{j(\omega t+\theta)}+e^{-j(\omega t+\theta)}}{2}\right|
$$

where $A(t)=e^{-\alpha t}, a$ is the relative amplitude and $\theta$ is the mode phase shift. $t$ is lying in a certain interval of time.

The exponential decay constant $\alpha$ and the angular frequency $\omega$ correspond to the real and the imaginary components, respectively. The Eigen values of the $i_{t h}$ mode expressed as $\lambda_{i}=\alpha_{i}+j \omega_{i}$. $f_{c}$ is the wavelet central frequency parameter and $f_{b}$ is a bandwidth parameter that controls the shape of the wavelet [8].

Representing $A(t)$ with its Taylor Series around the reference point $t=b$ returns: 
$A(t)=A(b)+\sum_{n=1}^{\infty}(t-b)^{n} \frac{A^{(n)}(b)}{n !}$

By substituting (8) into (5), the Complex Morlet CWT of $f(t)$ can be expressed as:

$$
W_{h} x(a, b)=\frac{1}{\sqrt{a}} \int_{-\infty}^{+\infty} A(t)\left[\frac{e^{j(\omega t+\theta)}+e^{-j(\omega t+\theta)}}{2}\right] h^{*}(t-b / a) d t
$$

Jumping to the result as derived in [7], in the form of time varying amplitude and phase angle the equation can be defined as:

$$
W_{h} x\left(a_{k}, t\right)=\frac{\sqrt{\pi a_{k} f_{b}} A e^{-\alpha t}}{2} e^{j(\omega t+\theta)}
$$

where $a_{k}=(2 \pi / \omega) f_{c}$ and $k$ is the corresponding mode to be analyzed. For the detail please refer to [7], [8]. The logarithmic and argument of the modulus $W_{h} x\left(a_{k}, t\right)$ can be written as (12) and (13) respectively:

$$
\begin{aligned}
& \ln \left|W_{h} x\left(a_{k}, t\right)\right|=-\alpha t+\ln \left(\frac{\sqrt{\pi a_{k} f_{b}} A e^{-\alpha t}}{2}\right) e^{j(\omega t+\theta)} \\
& \arg \left|W_{h} x\left(a_{k}, t\right)\right|=-\omega t+\theta
\end{aligned}
$$

The logarithmic decrement constant $\alpha$ and the phase decrement constant $\omega$ are extracted from (12) and (13) respectively.

The damping ratio is then calculated using:

$$
\xi=\frac{-\alpha}{\sqrt{a^{2}+\omega^{2}}}
$$

For the multi-mode signal with $m$ sets of data, the value of $\alpha$ and $\omega$ can be determined as in (15)\&(16).

$$
\begin{gathered}
\alpha=\frac{-m \sum_{i=1}^{m} b_{i} \ln \left|W_{h} x\left(a_{k}, b_{i}\right)\right|-\sum_{i=1}^{m} b_{i} \sum_{i=1}^{m} \ln \left|W_{h}\left(a_{k}, b_{i}\right)\right|}{m \sum_{i=1}^{m} b_{i}^{2}-\left(\sum_{i=1}^{m} b_{i}\right)^{2}} \\
\omega=\frac{-m \sum_{i=1}^{m} b_{i} \arg \left|W_{h} x\left(a_{k}, b_{i}\right)\right|-\sum_{i=1}^{m} b_{i} \sum_{i=1}^{m} \arg \left|W_{h}\left(a_{k}, b_{i}\right)\right|}{m \sum_{i=1}^{m} b_{i}^{2}-\left(\sum_{i=1}^{m} b_{i}\right)^{2}}
\end{gathered}
$$

Therefore, to dealing with the multimode signal, the Complex Morlet CWT is expressed as in (17) [7]:

$$
W_{h}\left(\sum_{i=1}^{N} x_{i}\right)(a, b)=\sum_{i=1}^{N} W_{h} x_{i}(a, b)
$$

where $N$ is the maximum number of modes in the signal. 


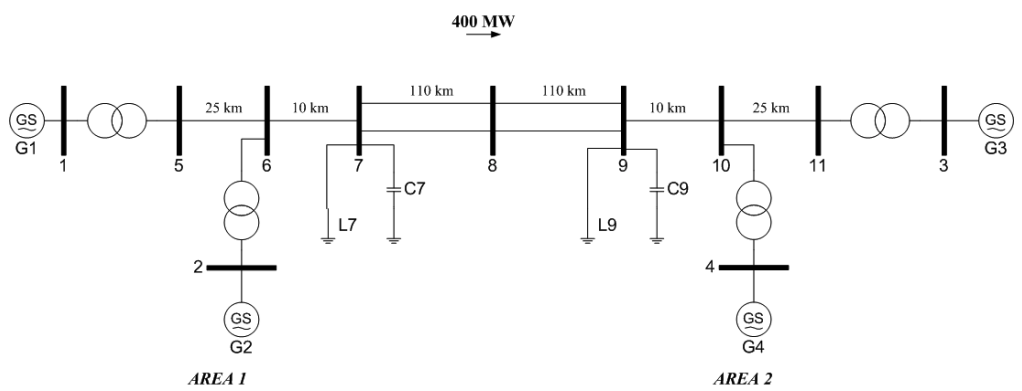

Fig. 4. Two area four machine system model.

\section{Experiment on the Two Area Four Machine System}

Fig. 4 shows a system consists of two identical areas interconnected by a weak tie line. The model is similar to the system in example-12.6 page-813 in [21]. A simulation of the small signal stability performance is carried out by applying an auto reclose action of the circuit breaker at the end one of a tie line from bus 7 to bus 8 in Fig. 4.

Exploiting this system, the authentication of the proposed method is elaborated. The percentage of load in L1 and L2 was increased gradually by 5 percent from 70 until 120 percent of initial value to see the relationship between load fluctuation and the damping ratio of inter-area oscillation that is shown in Fig. 5(a) and 5(b) respectively.

The calculation is attempted at every load scenario using PSAT toolbox developed by Prof F. Milano [22], which based on Eigen Value method.

Fig. 5(a) and Fig. 5(b) together depict the legitimacy of the proposed method based on the result of PSAT Toolbox. It is clearly shown the comparison between the result from the PSAT and the result calculated using the FFT-CWT method. This result confirms that the FFT-CWT method is robust enough to use in small signal stability assessment in a power system.

The distinction of this FFT-CWT method compare to other is that it does not need any information about system parameters; so that the calculation is purely rely on the signal captured from PMU.

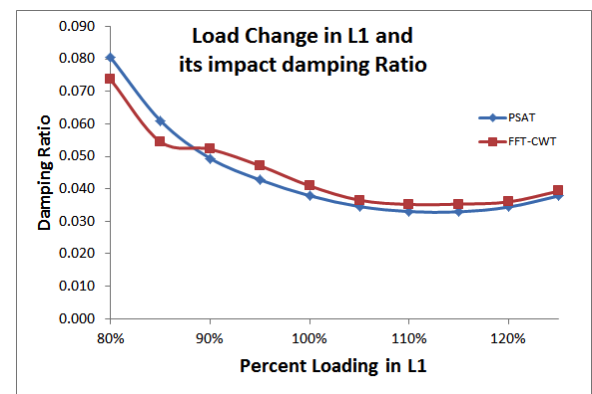

(a)

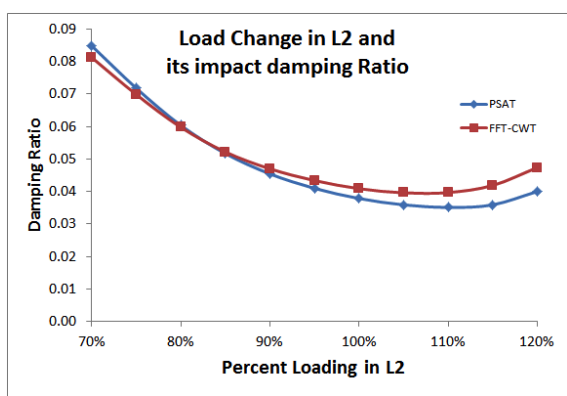

(b)

Fig. 5. Load Change vs. Damping ratio (a) Simulation in L1, (b) Simulation in L2.

\section{Case Study: Real PMU Data Analysis in Japan Campus WAMS}

Three significant system incidents occurred in Kyushu Electric Company generating unit that were recorded in PMU as released in the Website of Kyushu Electric Company (www.kyuden.co.jp). In this work, those events are assessed to observe the performance of the method when dealing with the real PMU Data.

\subsection{Kanda power plant unit-1 outage}

On $24^{\text {th }}$ of August 2003, around 17:50 pm. local time, Kanda Unit-1 was stop automatically due to a 
technical problem as released on $25^{\text {th }}$ of August 2003 in the company website (www.kyuden.co.jp/press_r_20030825_20030825_100001_1001.html). The phase difference signal measured between PMU in Kyushu Institute of Technology (KIT) and Nagoya Institute of Technology (NIT) and the corresponding center of oscillation are presented in Fig. 6(a) and Fig. 6(b) respectively. Logarithmic and phase decrement of the signal from the ridge of the oscillation are respectively shown in Fig. 7(a) and Fig. 7(b). Using linear regression analysis the value of $\alpha_{d}$ and $\omega_{d}$ are estimated and finally using (14) the damping ratio $(\xi)$ is estimated as 0.560 at the center of frequency oscillation $\left(f_{d}\right) 0.4525 \mathrm{~Hz}$.

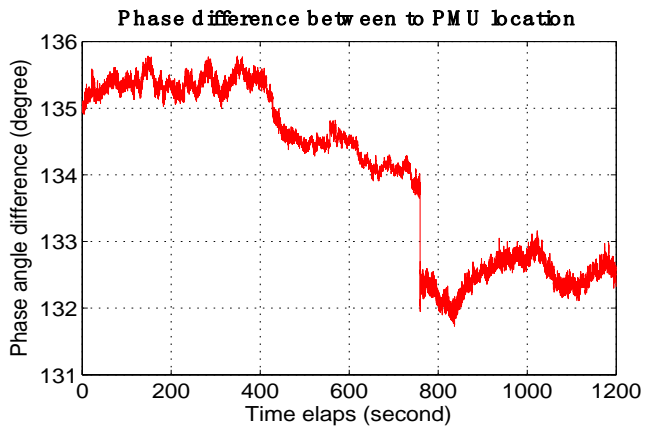

(a)

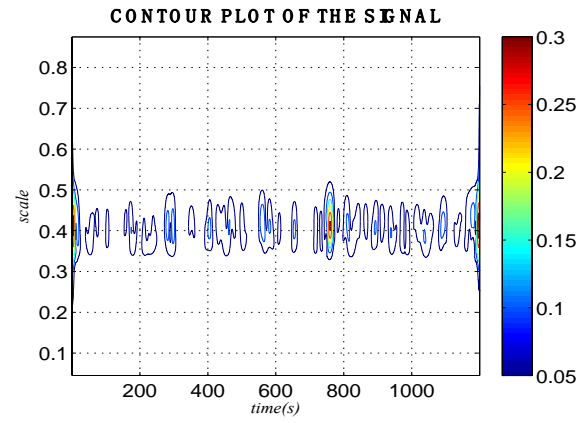

(b)

Fig. 6. Kanda\#1 shutdown on 24th -8-2003 (a) PMU signal from KIT to NIT, (b) Centre of Frequency.

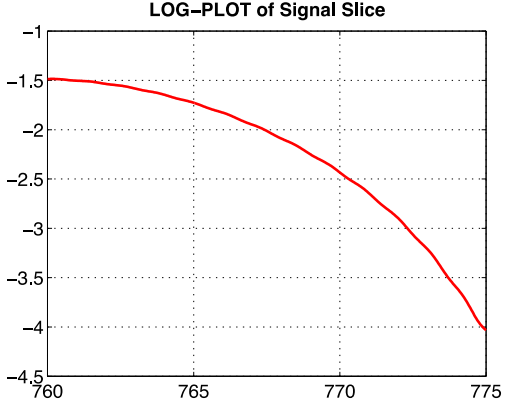

(a)

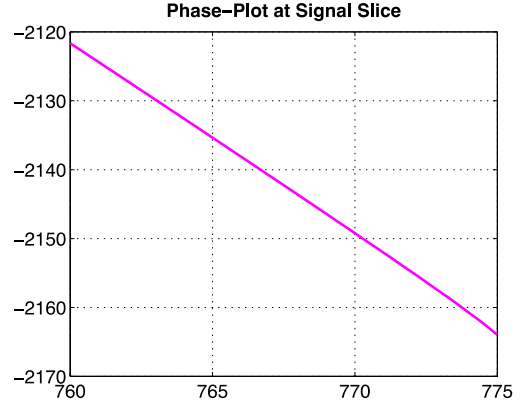

(b)

Fig. 7. (a). Logarithmic decrement and (b). Phase Decrement of the signal at $f_{c}=0.4525 \mathrm{~Hz}, f_{b}=25 \mathrm{~Hz}$ and scale $=0.1$ -20 .

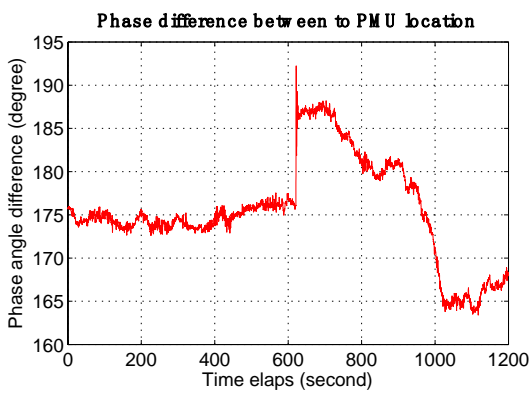

(a)

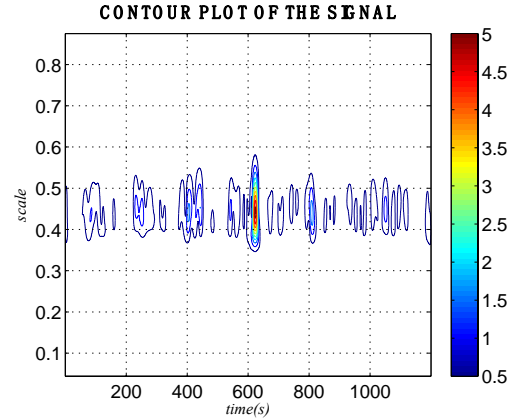

(b)

Fig. 8. Genkai\#4 shutdown on $4^{\text {th }}-10-2011$ (a). PMU signal from Miyazaki to NIT and (b). Centre of frequency.

\subsection{Genkai nuclear power unit-4 trip}

The next event was happened on $4^{\text {th }}$ of October 2011 when the Genkai nuclear power unit 4 (1.18GW) 
stopped automatically (www.kyuden.co.jp/press_111004-1.html). The data was recorded in PMU measurement and sent to Campus-WAMS server. The waveforms of phase difference measured between University of Miyazaki and Nagoya Institute of Technology (NIT) from 13:30 to 13:50 local time in $4^{\text {th }}$ of October 2011 is presented in Fig. 8.

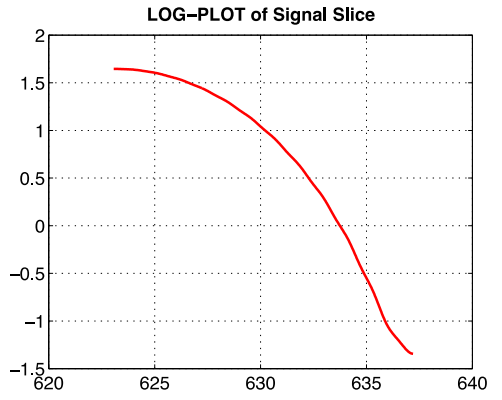

(a)

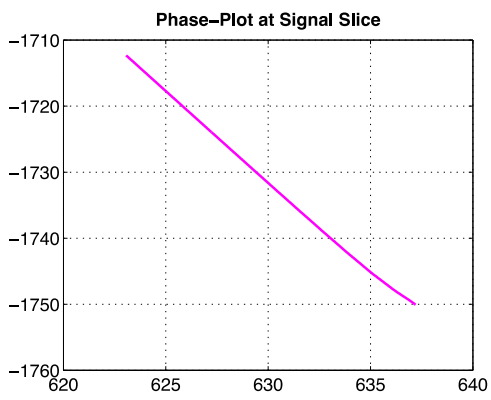

(b)

Fig. 9. (a). Logarithmic decrement and (b). Phase decrement of PMU signal from Miyazaki to NIT when Genkai\#4 shutdown on $4^{\text {th }}-10-2011$.

Damping ratio is calculated by demodulating the signal at the center of frequency oscillation. Then, the linear regression is applied to the demodulated signal. Fig. 9(a) and Fig. 9(b) respectively show the logarithmic and phase decrement of the signal from the ridge of the oscillation until the oscillations disappear after some seconds. Again, using linear regression analysis the value of $\alpha_{\mathrm{d}}$ and $\omega_{\mathrm{d}}$ are calculated. Damping ratio is estimated as 0.08 at center of oscillation frequency $f_{\mathrm{d}}=0.4409 \mathrm{~Hz}$.

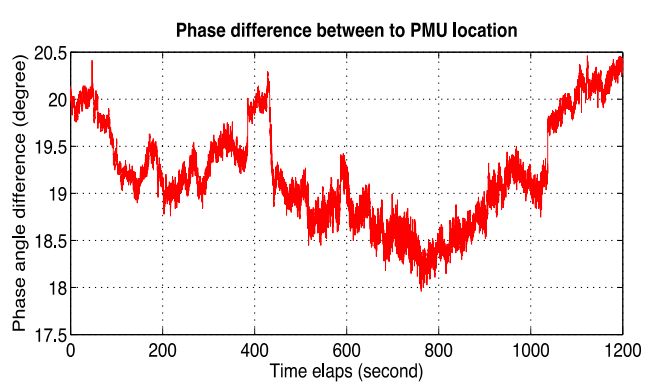

(a)

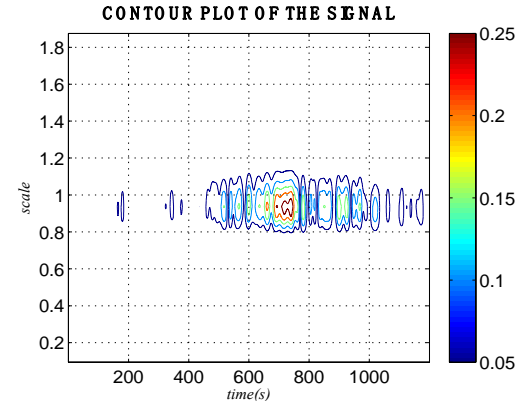

(b)

Fig. 10. Kanda\#2 shutdown on $27^{\text {th }}-8-2014$ (a) PMU signal from KIT to NIT and (b) Centre of frequency.

\subsection{Kanda unit-2 shutdown on August 2014}

On $27^{\text {th }}$ of August 2014, it was around 17:50 pm, Kanda unit-2 of Kyushu Electric Company (375 MW capacity, operates at $150 \mathrm{MW}$ ) shutdown after a steam leakage was found on 15:50, two hours prior to the unit shutdown (www.kyuden.co.jp/press h140827b-1.html). The PMU measurement between KIT and NIT is shown in Fig. 10. Using a same manner as previous, the center frequency oscillation is estimated at $0.1708 \mathrm{~Hz}$ during the occurrence, which means no forced inter-area oscillation was detected. The oscillation in this situation is just an ambient oscillation that normally occurs on normal operation. Fig. 10 also indicates that there was no any sudden change in the phase differences pattern between two PMU locations that might because of the shutdown was well prepared therefore was no large impact to the system stability.

In subsection 5.2 we can find the damping ratio is very low (0.08) compare to the damping ration in subsection 5.1 that is 0.560 . It can be understood since the capacity of outage unit in 5.2 was much higher than the other one. At the third occasions (section 5.3), the center of oscillation is only $0.1708 \mathrm{~Hz}$ hence no forced inter-area oscillation was detected as the effect of the disturbance. 


\section{Conclusion}

This FFT-CWT approach is accountable enough to identify inter area oscillation mode in the system as well as to calculate the damping ratio based on the information extracted from the PMU data without knowing the parameters of the system. The validity of this method was verified by comparing to the result of the PSAT calculation. It was demonstrated that the results of the two approaches confirmed each other, means that this FFT-CWT is feasible enough to estimate the damping ratio of a small signal oscillation in a power system. The novelty of this method is that system parameter are not involved in the calculation process. In addition, FFT has been efficiently utilized to determine the center of the oscillation as one of the wavelet parameters.

\section{References}

[1] Wilson R, Sterlina P. Verification of measured transmission system phase angles. IEEE Transactions on Power Delivery, 1996; 11(4):1743-1747.

[2] Phadke A, Kasztenny B. Synchronized phasor and frequency measurement under transient conditions. IEEE Transactions on Power Delivery, 2009;24(1):89-95.

[3] Phadke A, Thorp J. History and applications of phasor measurements. In: Proc. of Power Systems Conference and Exposition, 2006:331-335.

[4] Premerlani W, Kasztenny B, Adamiak M. Development and implementation of a synchrophasor estimator capable of measurements under dynamic conditions. IEEE Transactions on Power Delivery, 2008;23(1):109-123.

[5] Yao Z. Fundamental phasor calculation with short delay. IEEE Transactions on Power Delivery, 2008;23(3):1280-1287.

[6] Hur K, Santoso S. Estimation of system damping parameters using analytic wavelet transforms. IEEE Transactions on Power Delivery, 2009;24(3):1302-1309.

[7] Rueda J, Juarez C, Erlich I. Wavelet-based analysis of power system low-frequency electromechanical oscillations. IEEE Transactions on Power System, 2011;26(3):1733-1743.

[8] Rueda J, Erlich I. Enhanced wavelet-based method for modal identification from power system ringdowns. In: Proc. of PowerTech 2011 IEEE Trondheim, 2011:1-8.

[9] Staszewski W. Identification of damping in mdof systems using time-scale decomposition. Journal of Sound and Vibration, 1997;23(2):283-305.

[10] Slavic^ J, Simonovski I, Boltez^ar M. Damping identification using a continuous wavelet transform: Application to real data. Journal of Sound and Vibration, 2003;262(2):291-307.

[11] Ngamroo I, Danupaprita S, Mitani Y. PMU based monitoring of inter-area oscillation in Thailand power system via home power outlets. ECTI Transaction on Electrical Engineering, Electronics and Communication, 2007;5(2):199-204.

[12] Ota Y, Hashiguchi T, Ukai H, Sonoda M, Miwa Y, Takeuchi A. Monitoring of interconnected power system parameters using PMU based WAMS. In: Proc. of Power Tech, 2007 IEEE Lausanne, 2007:1718- 1722.

[13] Ren J, Kezunovic M. Real-time power system frequency and phasors estimation using recursive wavelet transform. IEEE Transactions on Power Delivery, 2011;26(3):1392-1402.

[14] Nam SR, Young Park J, Kang SH, Kezunovic M. Phasor estimation in the presence of dc offset and ct saturation. IEEE Transactions on Power Delivery, 2009;24(4):1842-1849.

[15] Hashiguchi T, Watanabe M, Matsushita A, Mitani Y, Saeki O, Tsuji K, Hojo M, Ukai H. Identification of characterization factor for power system oscillation based on multiple synchronized phasor measurements. Electrical Engineering in Japan, 2008;163(3):10-18.

[16] Brigham EO. The Fast Fourier Transform and Its Applications. Englewood Cliffs, NJ: Prentice Hall; 1988.

[17] Tarasiuk T. Hybrid wavelet-fourier spectrum analysis. IEEE Transactions on Power Delivery, 2004;19(3):957-964.

[18] Browne T, Vittal V, Heydt G, Messina A. A comparative assessment of two techniques for modal identification from power system measurements, IEEE Transactions on Power System, 2008;23(3):1408-1415.

[19] Mallat S. A Wavelet Tour of Signal Processing, 2nd ed. San Diego: Academic Press; 1999.

[20] Lin T, Domijan A. Recursive algorithm for real-time measurement of electrical variables in power systems. IEEE Transactions on Power Delivery, 2006;21(1):15-22.

[21] Kundur P, Balu NJ, Lauby MG. Power System Stability and Control, EPRI Power System Engineering Series. New York: McGraw-Hill; 1994.

[22] Milano F. An open source power system analysis toolbox. In: Proc. of IEEE Power Engineering Society General Meeting, 2006. 